\title{
Neural Mechanisms of Learning are Critically Dependent on Sleep
}

Anna á V. Guttesen ${ }^{1}$, M. Gareth Gaskell ${ }^{1,2}$, Emily V. Madden ${ }^{1}$, Gabrielle Appleby ${ }^{1}$, Zachariah R. Cross ${ }^{3}$, Scott A. Cairney ${ }^{1,2 *}$

${ }^{1}$ Department of Psychology, University of York, York, UK

${ }^{2}$ York Biomedical Research Institute, University of York, York, UK

${ }^{3}$ Cognitive and Systems Neuroscience Research Hub, University of South Australia, Adelaide, Australia

*Corresponding author 


\begin{abstract}
Sleep supports memory consolidation as well as next-day learning. The Active Systems account of offline consolidation suggests that sleep-associated memory processing paves the way for new learning, but empirical evidence in support of this idea is scarce. Using a withinsubjects, crossover design, we assessed behavioural and electrophysiological indices of episodic encoding after a night of sleep or total sleep deprivation in healthy adult humans (aged 18-25 years), and investigated whether the behavioural measures were predicted by the overnight consolidation of episodic associations formed the previous day. Sleep supported memory consolidation and next-day learning, as compared to sleep deprivation. However, the magnitude of this sleep-associated consolidation benefit did not significantly predict the ability to form novel memories after sleep. Interestingly, sleep deprivation prompted a qualitative change in the neural signature of encoding: whereas $12-20 \mathrm{~Hz}$ beta desynchronization - an established EEG marker of successful encoding - was observed after sleep, sleep deprivation disrupted beta desynchrony during successful learning. Taken together, our findings suggest that effective learning mechanisms are critically dependent on sleep, but not necessarily sleep-associated consolidation.
\end{abstract}




\section{Introduction}

How do we remember events from days gone by? It is now firmly established that sleep facilitates memory consolidation; the process by which weak and initially labile memory traces become strong and enduring representations (Gais et al., 2006; Payne et al., 2012; Talamini et al., 2008; Ashton et al., 2020; Gaskell et al., 2018; Cairney et al., 2018a; Durrant et al., 2016; Ashton \& Cairney, 2021). Whereas sleep was originally thought to provide passive protection to consolidation (i.e. by shielding memories from the interference posed by wakeful experience), recent work suggests that newly formed memories are actively strengthened during sleep (Rasch et al., 2007; Cairney et al., 2018b; Wang et al., 2019; Schreiner et al., 2021; Schonauer et al., 2017). The Active Systems account of sleep-associated consolidation posits that a reactivation of hippocampus-dependent memories during slow-wave sleep (SWS) facilitates the migration of hippocampus-dependent memory to neocortex for long-term storage (Born \& Wilhelm, 2012; Rasch \& Born, 2013; Klinzing et al., 2019).

While the benefits of sleep for consolidation are well known, recent work has indicated that sleep also supports next-day learning in hippocampus. When a night of total sleep deprivation precedes a novel learning opportunity, declarative memory recall is severely impaired, even after recovery sleep, suggesting that sleep loss disrupts hippocampal encoding (Tempesta et al., 2016; Alberca-Reina et al., 2014; Kaida et al., 2015; Cousins et al., 2018). Indeed, as compared to a normal night of sleep, sleep deprivation weakens hippocampal responses during subsequent learning, leading to a significant decline in recall performance several days later (Yoo et al., 2007). Correspondingly, daytime naps not only benefit learning (Mander et al., 2011), but also restore hippocampal encoding capabilities relative to an equivalent period of wakefulness (Ong et al., 2020).

Intriguingly, the benefits of sleep for consolidation and next-day learning appear to depend on overlapping mechanisms. Slow oscillations $(<1 \mathrm{~Hz})$, which are a prominent feature of SWS, have been causally linked to overnight memory retention (Ngo et al., 2013; Perl et al., 2016; Leminen et al., 2017; Ong et al., 2016; Papalambros et al., 2017; Marshall et al., 2006), and are thought to play a central role in the reactivation and reorganisation of hippocampusdependent memories (Born \& Wilhelm, 2012; Rasch \& Born, 2013; Klinzing et al., 2019). Slow oscillations also support subsequent learning in hippocampus: using non-invasive auditory interventions to enhance or disrupt slow oscillation activity $(\mathrm{SOA}, 0.5-4 \mathrm{~Hz}$ ) respectively improves or impairs declarative memory encoding the following day (Antonenko et al., 2013; 
Ong et al., 2018; Van der Werf et al., 2009). This seemingly holistic memory function of slow oscillations raises important questions about the relationship between sleep-associated consolidation and next-day learning. From an Active Systems perspective, slow oscillations orchestrating the migration of declarative memories from hippocampus to neocortex might equally facilitate new encoding in hippocampus. Furthermore, if there is a common resource in sleep that facilitates both consolidation and encoding, then one should see an association across individuals between the strength of that resource, the strength of overnight consolidation and the strength of subsequent learning.

In this preregistered study (osf.io/78dja), we tested the hypothesis that the extent to which individuals consolidate new memories during sleep would predict their ability to encode novel information the following day, and that SOA would contribute to this relationship. In a withinsubjects, crossover design (Figure 1), healthy young adults were trained on a visuospatial memory task before a night of either EEG-monitored sleep or total sleep deprivation, and were tested the following morning. Afterwards, participants were trained on a novel pairedassociates task, but were not tested until two days later (allowing for recovery sleep in the sleep deprivation condition). Retrieval performance on the visuospatial memory and pairedassociates tests thus provided independent metrics of overnight consolidation and next-day learning, respectively. Importantly, because both tasks probed associative memory, which relies on hippocampus (for reviews, Eichenbaum 2004; Konkel \& Cohen, 2009), we could target the relationship between sleep-associated consolidation and subsequent encoding of hippocampus-dependent memories.

We compared overnight sleep and sleep deprivation so that we could also investigate how protracted sleep loss affects the neurocognitive mechanisms of learning. Specifically, EEG recordings were acquired during paired-associates learning to test the hypothesis that sleep deprivation would disrupt theta $(4-8 \mathrm{~Hz})$ and gamma $(>40 \mathrm{~Hz})$ synchronisation, which support item binding in episodic memory (Köster et al., 2018; Summerfield \& Mangels, 2005; Henin et al., 2019). Furthermore, in exploratory analyses, we investigated the effect of sleep deprivation on $12-20 \mathrm{~Hz}$ beta desynchronization; an established marker of successful learning that reflects depth of information processing (HansImayr et al., 2009; 2011; 2012; Griffiths et al., 2019). Understanding the mechanisms by which sleep disturbances impair learning and memory is increasingly important in modern society, where many people fail to regularly 
obtain an adequate amount of sleep (Bonnet \& Arand, 1995; Stranges et al., 2012; Becker et al., 2018).

a)

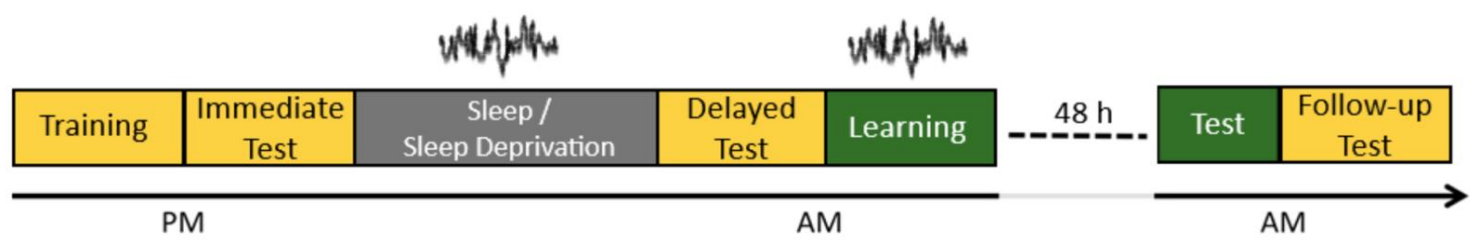

b)

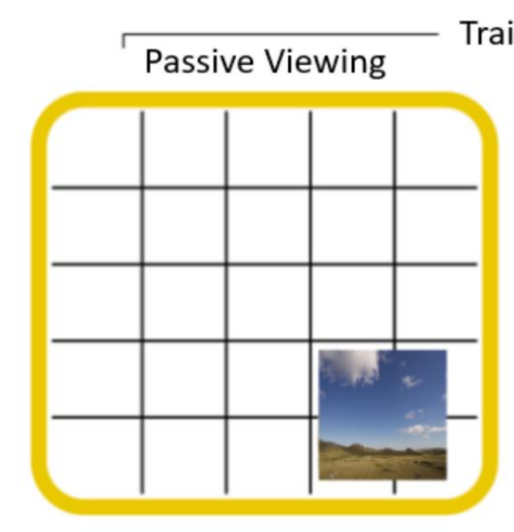

raining

Active Viewing

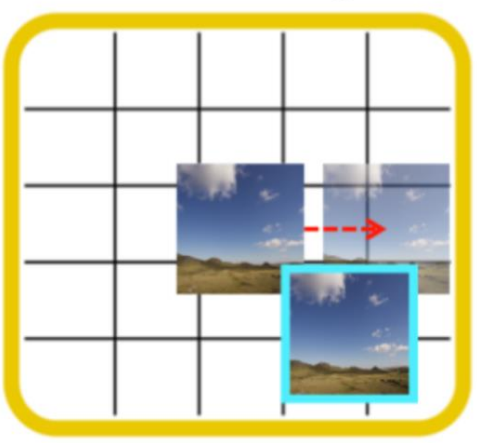

Test

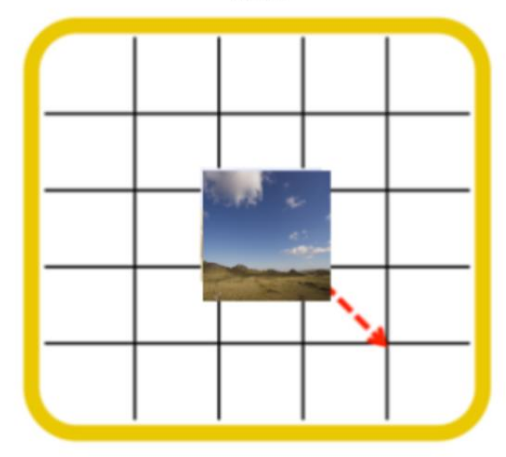

c)
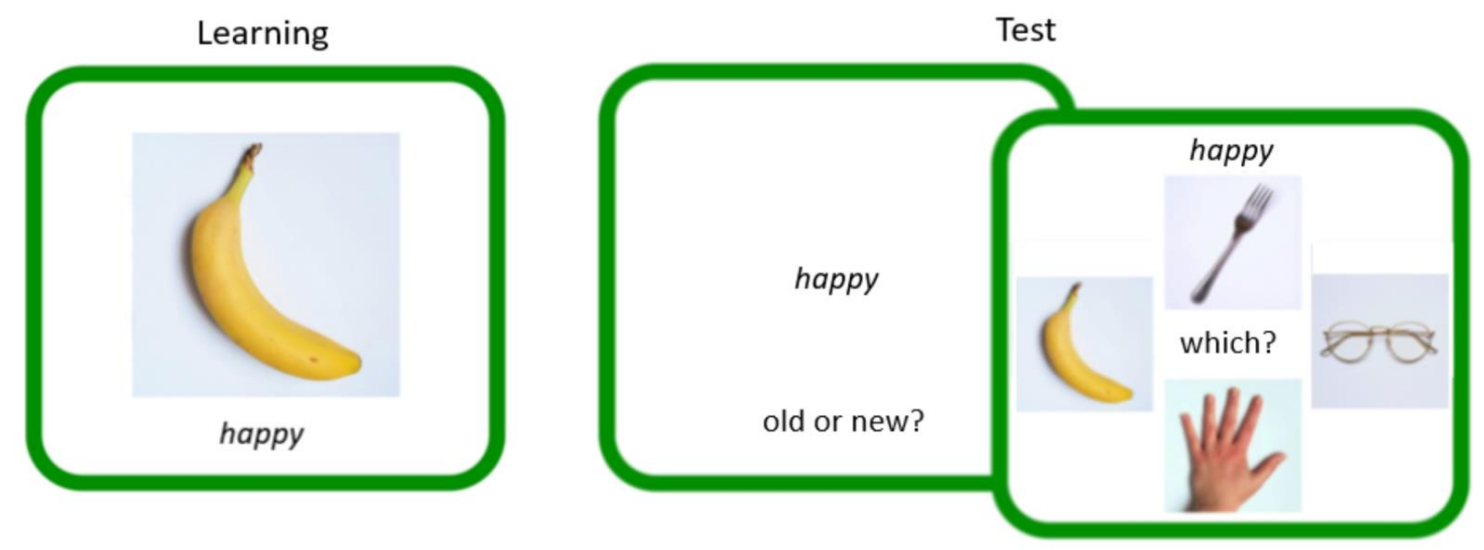

Figure 1. Experimental procedures and tasks.

a) Study timeline. The colours represent the tasks: visuospatial task in yellow (see b) and paired-associates task in green (see c). Participants arrived in the evening (8:30 PM - 10 PM) to complete the visuospatial task (training and immediate test). After overnight sleep or sleep deprivation (7:00 AM - 8:30 AM), participants were tested again (delayed test) and then completed the learning phase of the paired-associates task. $48 \mathrm{~h}$ later (after recovery sleep), participants returned to complete the paired-associates test and a follow-up visuospatial memory test. EEG recordings were acquired during sleep and paired-associates learning. 
b) Visuospatial memory task. Participants completed one round of passive viewing, during which they viewed the location of each image on a grid. Next, in the active viewing phase, participants moved each image to the location that they thought it had appeared during passive viewing and received feedback on its correct location (bright blue frame). Active viewing continued until participants had met the performance criterion for all images $(<4.8 \mathrm{~cm}$ from correct location, mean \pm SD number of rounds to meet criterion: Sleep: $8.77 \pm 2.39$, Sleep Deprivation: $9.07 \pm 2.89$ ). The test phases followed the same procedures as one round of active viewing, but no feedback was provided. Each test trial provided an accuracy score in $\mathrm{cm}$, which described how far the image was placed from its correct location.

c) Paired-associates task. Participants completed one round of learning, during which they encoded adjective-image pairings. At test, a word was presented in isolation and participants first indicated whether it was 'old' (i.e. they recognised the word from learning) or 'new' (i.e. they did not recognise the word from learning). For correctly recognised (old) words, participants then indicated which of the four presented images was associated with that word at learning. For words identified as new, or for previously unseen words that were incorrectly identified as old, participants moved immediately onto the next trial.

\section{Results}

All participants $(N=30,17$ females, mean $\pm S D$ age, $20.10 \pm 1.65)$ took part in both experimental conditions (Sleep and Sleep Deprivation, Figure 1a). Conditions were separated by at least one week and condition order was counterbalanced. Performance on the visuospatial (Figure 1b) and paired-associates tasks (Figure 1c) provided independent metrics of overnight consolidation and next-day learning, respectively.

\section{Sleep facilitates overnight consolidation}

To assess overnight consolidation, we computed a retention index (RI) from the visuospatial tests: the error score for each image (i.e. distance in $\mathrm{cm}$ from its correct location) at the delayed test was subtracted from the error score at the immediate test, and the difference was averaged over trials (higher RI = better retention). As expected, RI was significantly higher after sleep than after sleep deprivation $(t(28)=3.78, p<.001, d=0.70$, Figure $2 a)$. To ensure that our findings were not driven by between-condition differences in fatigue at the delayed test, we also assessed memory retention between the immediate and follow-up test (which took place $48 \mathrm{~h}$ after the delayed test, thereby allowing for recovery sleep). As expected, the RI was 
still significantly higher in the sleep (vs sleep deprivation) condition $(t(28)=2.18, p=.038$, $d=0.41$, Figure $2 b$ ), confirming that sleep had facilitated overnight consolidation. There were no significant between-condition differences in recall accuracy at the immediate test (mean \pm SE, sleep: $2.44 \pm 0.10$, sleep deprivation: $2.57 \pm 0.10, t(28)=-0.98, p=.337, d=0.18)$.

\section{Sleep improves next-day learning}

To assess encoding capabilities after sleep or sleep deprivation, we calculated a learning index (LI), which equated to the percentage of correctly recognised images on the paired-associates test. Because this test took place $48 \mathrm{~h}$ after paired-associates learning (thereby allowing for recovery sleep), any between-group difference in performance cannot be explained by fatigue. As expected, learning performance was significantly higher after sleep than after sleep deprivation $(t(29)=12.19, p<.001, d=2.23$, Figure $2 c)$, indicating that sleep had improved next-day learning. Although response times on a psychomotor vigilance task (PVT, see Methods) were slower in the morning after sleep deprivation (mean $\pm \mathrm{SE}, 399.00 \mathrm{~ms} \pm 17.63$ ) than sleep $(289.15 \pm 4.34, p<.001)$, an exploratory correlation showed no significant relationship between $\mathrm{LI}^{\text {SleepBenefit }}$ (i.e. [sleep condition $\mathrm{LI}$ - sleep deprivation condition LI], see below) and $\mathrm{PVT}^{\text {SleepBenefit }}$ (i.e. [mean RT after sleep - mean RT after sleep deprivation], $\mathrm{R}^{2}=-.30$, $\mathrm{p}=.113$ ). Similarly, as indicated by the Stanford Sleepiness Scale (SSS), participants felt less alert after sleep deprivation (mean $\pm \mathrm{SE}, 5.37 \pm 0.15)$ than sleep $(2.27 \pm 0.16)$. However, there was no significant correlation between $\mathrm{LI}^{\text {SleepBenefit }}$ and SSS ${ }^{\text {SleepBenefit }}$ (i.e. [mean rating after sleep - mean rating after sleep deprivation], $\mathrm{R}^{2}=-.35, \mathrm{p}=.056$ ). Extended analysis of the PVT and Stanford Sleepiness Scale data is available in the Supplementary Results. 
a)

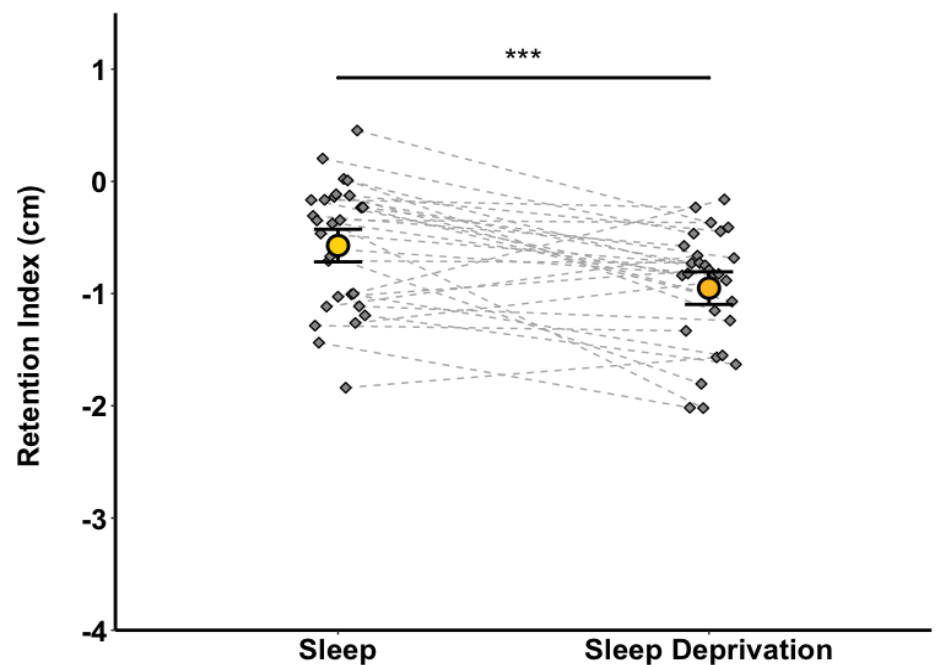

b)

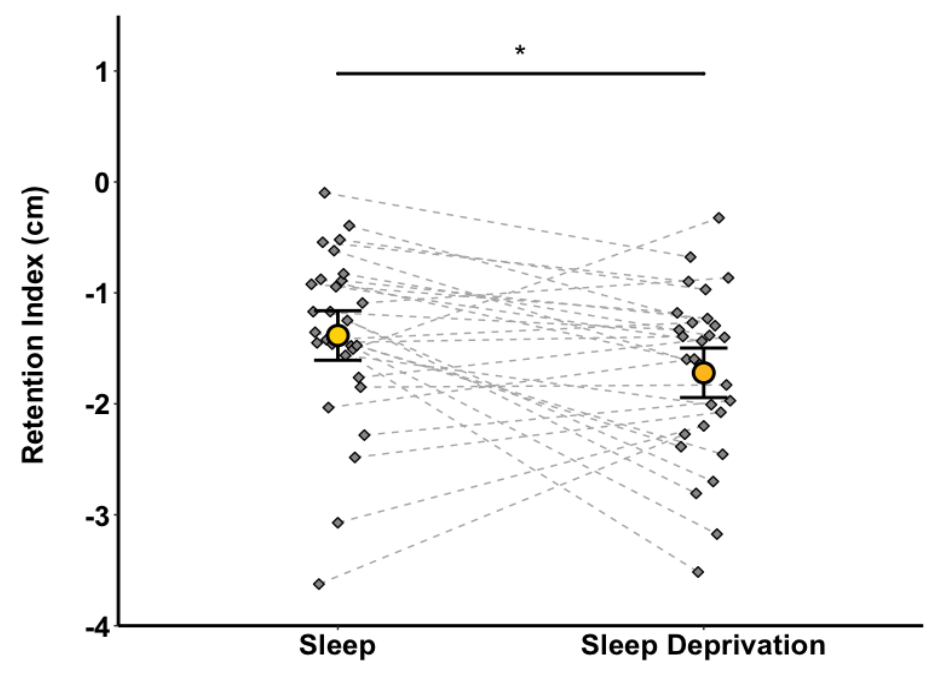

c)

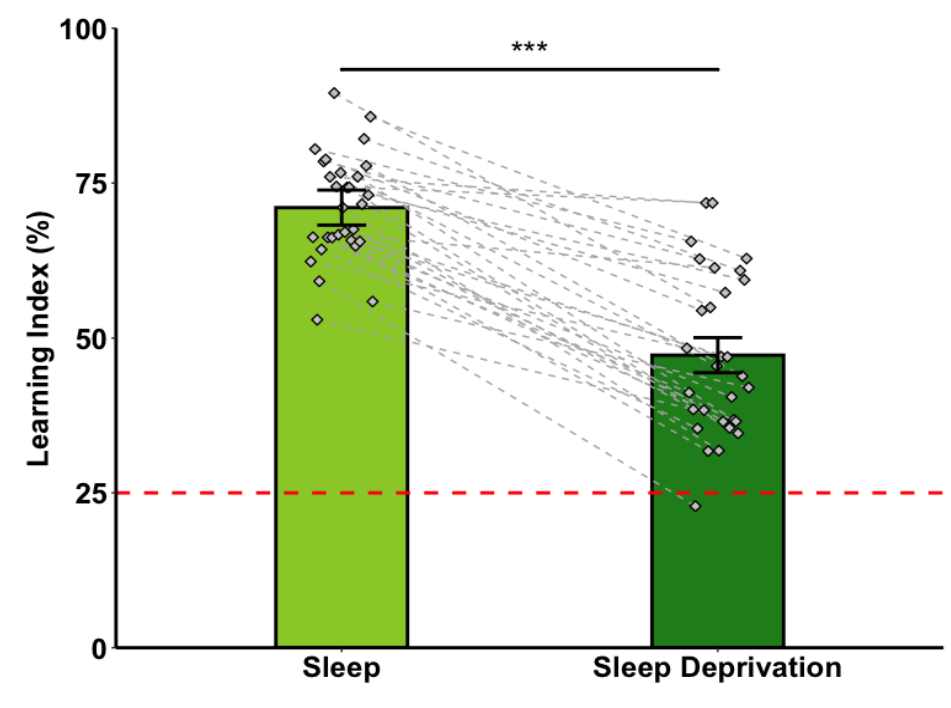

Figure 2. Behaviour.

a) Retention index (RI) between the immediate and delayed test, and b) between the immediate and the follow-up test (i.e. $48 \mathrm{~h}$ after the delayed test, thereby allowing for recovery sleep). A higher $\mathrm{RI}$ indicates better retention. c) Learning index (LI, tested $48 \mathrm{~h}$ after sleep or sleep deprivation). Higher scores indicate better learning and the red-dotted line represents chance performance. All figures show group means ( \pm SEM). Diamonds and connecting lines represent individual participants. ${ }^{* *} p<.001, * p<.05$. 


\section{No relationship between sleep-associated consolidation, slow oscillation activity and next- day learning}

Next, we tested the hypothesis that overnight consolidation predicts next-day learning, and that slow oscillation activity (SOA) contributes to this relationship. Because our aim was to target the relationship between sleep-associated memory processing and next-day learning, it was necessary to first quantify the positive impact of sleep (vs sleep deprivation) on the RI and LI. We therefore subtracted both the RI and LI between the sleep and sleep deprivation conditions (on a participant-by-participant basis), such that positive scores on the resultant $\mathrm{RI}^{\text {SleepBenefit }}$ and $\mathrm{LI}^{\text {sleepBenefit }}$ metrics indicated a sleep-associated improvement in performance. SOA was defined as EEG power within the $0.5-4 \mathrm{~Hz}$ frequency band during sleep stages N2 and slow-wave sleep (collapsed across all EEG channels). In a multiple regression model, we entered $\mathrm{RI}^{\text {SleepBenefit }}$ and SOA as predictors and $\mathrm{LI}^{\text {SleepBenefit }}$ as the outcome variable. Contrary to expectations, sleep-associated consolidation ( $\mathrm{RI}^{\text {SleepBenefit }}$ ) and SOA did not significantly account for next-day learning ( $\left(\left.\right|^{\text {SleepBenefit }}, F(2,24)=1.51, R^{2}=0.11, p=.242\right.$, Figure 3 ). No significant relationship was observed between $\mathrm{RI}^{\text {sleepBenefit }}$ and $\mathrm{LI}^{\text {sleepBenefit }}$ independently of SOA $(B=3.30, t(24)=0.86, p=.399)$, nor between SOA and $L^{\text {SleepBenefit }}$ independently of RI ${ }^{\text {sleepBenefit }}(B=-$ $0.51, t(24)=-1.65, p=.111)$. RI ${ }^{\text {sleepBenefit }}$ did not significantly correlate with SOA $\left(R^{2}=0.21\right.$, $p=.298$ ). A follow-up Bayesian analysis revealed anecdotal evidence in support of the null (i.e. that sleep-associated consolidation and SOA did not account for next-day learning, $\mathrm{BF}_{01}=2.04$ ). In a subsidiary analysis, we repeated this multiple regression but only entered data from the sleep condition into our model (i.e. the $\mathrm{RI}^{\text {SleepBenefit }}$ and $\mathrm{LI}^{\text {SleepBenefit }}$ were replaced with the $\mathrm{RI}$ and LI from the sleep condition alone). Our findings mirrored those of the foregoing analysis: sleep-associated consolidation (RI) and SOA did not significantly account for next-day learning (LI, $\left.F(2,25)=1.83, p=.181, R^{2}=0.13, B F_{01}=1.68\right)$. There was also no significant relationship between $\mathrm{RI}$ and $\mathrm{LI}$ independently of SOA $(B=4.46, t(25)=1.67, p=.107)$, nor between SOA and $\mathrm{LI}$ independently of $\mathrm{RI}(\mathrm{B}=-0.25, \mathrm{t}(25)=-1.16, \mathrm{p}=.256)$, and no significant correlation was observed between $\mathrm{RI}$ and SOA $\left(\mathrm{R}^{2}=0.28, \mathrm{p}=.143\right)$.

We also explored whether RI in the sleep condition was correlated with other sleep parameters previously implicated in declarative memory consolidation: time $(\mathrm{min})$ in slowwave sleep (Scullin, 2013; Backhaus et al., 2006) and fast spindle power (12.1-16 Hz, Tamminen et al., 2010; Cox \& Talamini, 2012). However, no significant relationships emerged (all $p>$.36). 


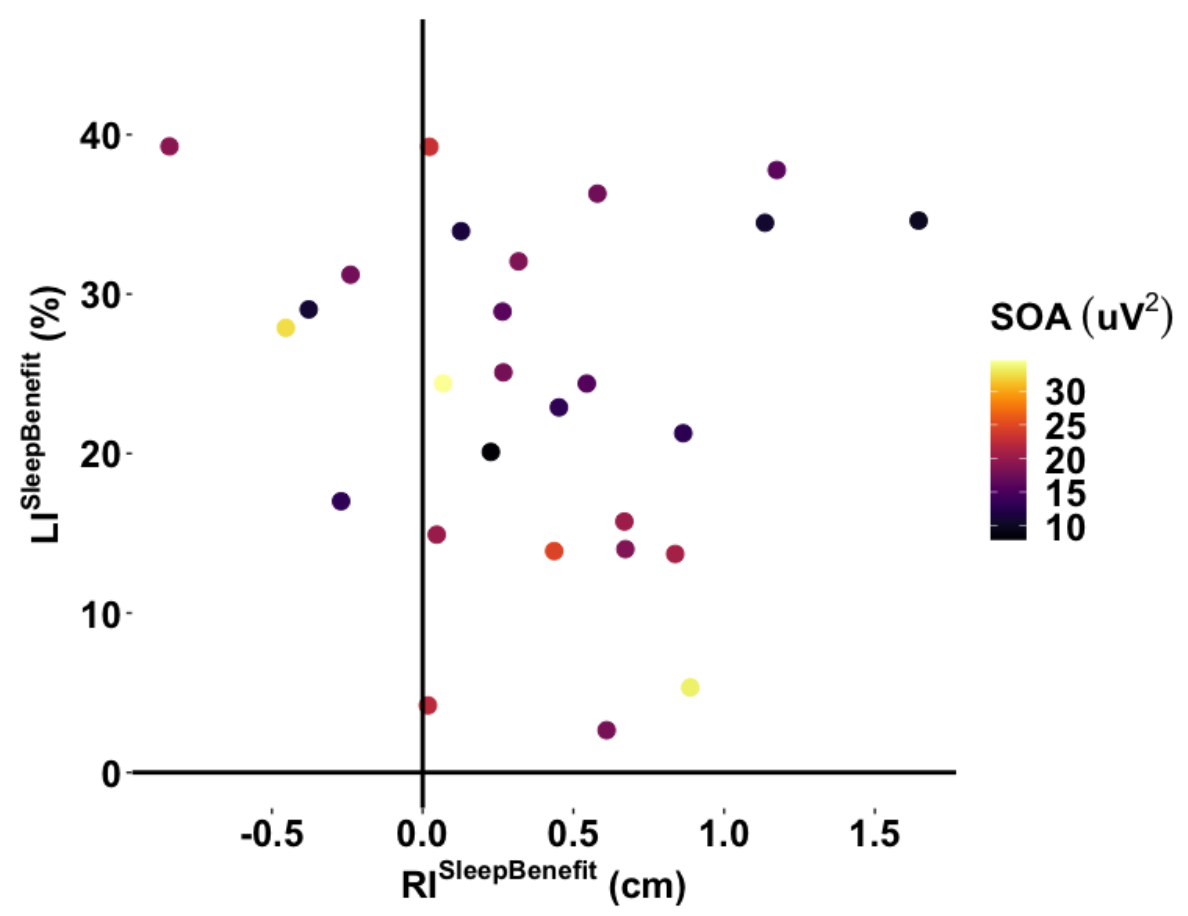

Figure 3. Relationship between sleep-associated consolidation, SOA and next-day learning. Sleep-associated consolidation ( $\mathrm{RI}^{\text {sleepBenefit }}$ and SOA had no significant impact on next-day learning (LI SleepBenefit $^{\text {. }}$.

\section{Sleep deprivation disrupts beta desynchronization during successful learning}

We explored the effect of sleep deprivation on EEG beta desynchronization; an established marker of successful encoding (Griffiths et al., 2019; HansImayr et al., 2009, 2011). Consistent with previous findings, subsequently remembered (vs. forgotten) adjective-image pairings were associated with a significant decrease in beta power at learning (corresponding to two clusters in the left hemisphere beginning at $\sim 1.5 \mathrm{~s}(p=.044, d=-.66)$ and $\sim 1.75 \mathrm{~s}(p=.038, d=-$ .49) after stimulus onset (Figure 4A). Interestingly, changes in beta power accompanying successful learning were significantly different in the sleep and sleep deprivation conditions (interaction, corresponding to a cluster in the left hemisphere at $\sim 0.5-0.7 \mathrm{~s}, p=.014, d=-.33$, Figure $4 B$ and $4 D$ ). Whereas subsequently remembered (vs forgotten) adjective-image pairings were associated with beta desynchronization after sleep ( $p=.005)$, an apparent upregulation of beta power emerged from the same contrast after sleep deprivation $(p=.019$, Figure 4C, although this latter post-hoc test did not survive Bonferroni correction, alpha = .0125). There was also a significant downregulation of beta power for subsequently remembered pairings in the sleep (vs sleep deprivation) condition, $(p=.001)$, but no such between-condition difference for subsequently forgotten pairings ( $p=.928)$. A significant 
overall reduction in beta power was observed in the sleep (vs. sleep deprivation) condition when combining subsequently remembered and forgotten adjective-image pairings, corresponding to two clusters in the left $(p=.014, d=-0.63)$ and right hemisphere $(p=.038, d$ $=-0.47)$.

No significant differences were observed in the preregistered theta $(4-8 \mathrm{~Hz})$ or gamma $(40-60$ $\mathrm{Hz}$ ) bands for subsequently remembered $>$ forgotten adjective image pairings, sleep $>$ sleep deprivation or the interaction between these contrasts (all $p$ >.142). Given the previously reported links between alpha $(8-12 \mathrm{~Hz}$ ) desynchronization and successful learning (Griffiths et al., 2019; 2021; Weisz et al., 2020), we also explored activity in this frequency band (same contrasts as above), but no significant effects were observed (all $p>.396$ ). 
a)

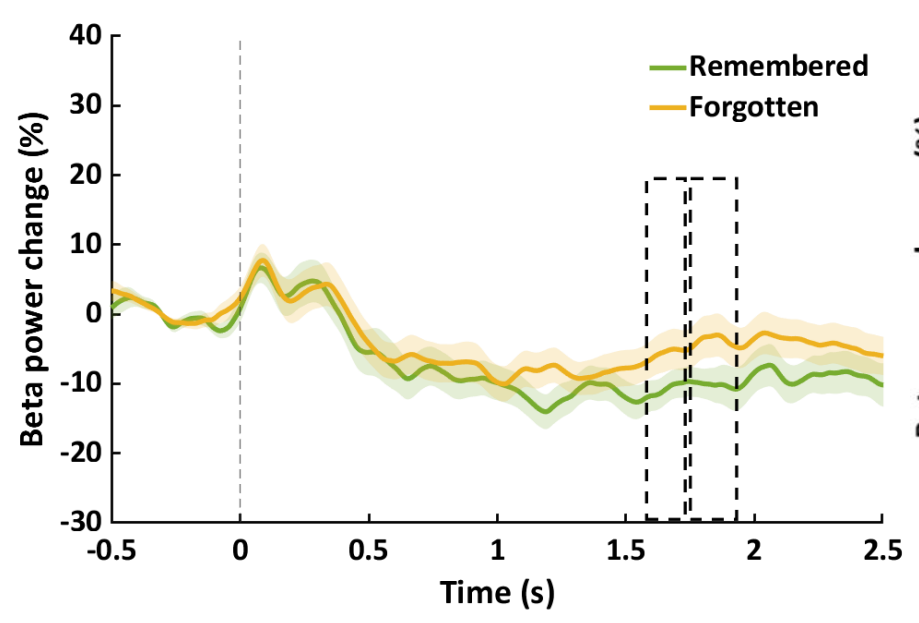

c)

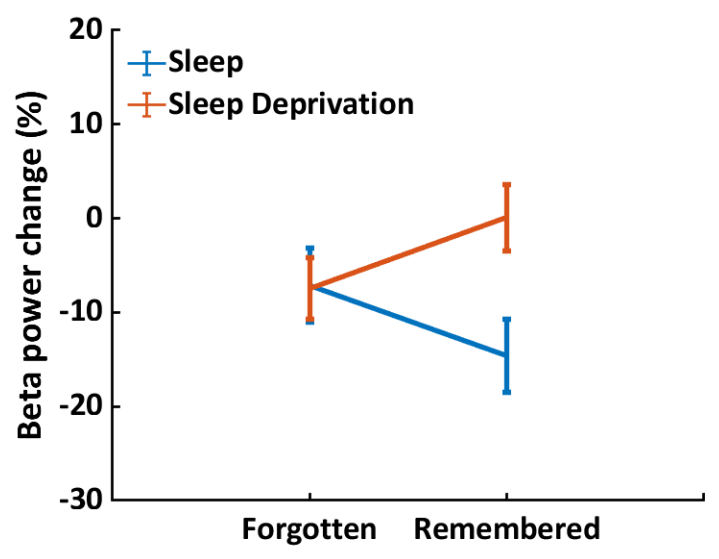

b)

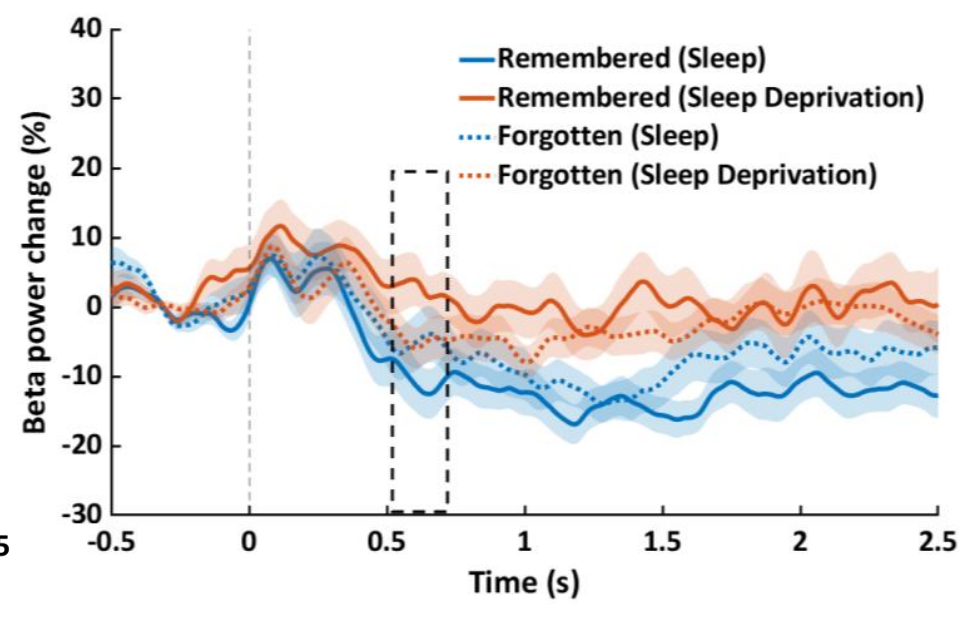

d)

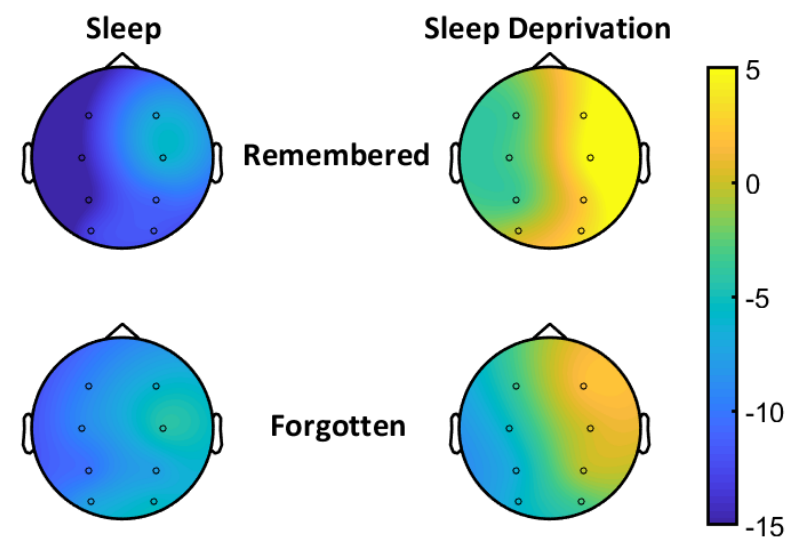

Figure 4. Changes in beta power during successful learning after sleep and sleep deprivation

a) Grand average change in beta power from baseline $(12-20 \mathrm{~Hz}$, all channels) for subsequently remembered and forgotten adjective-image pairings. b) The same contrast presented separately for the sleep and sleep deprivation conditions. Dashed boxes approximate the time windows contributing to the identified clusters and shaded areas represent the standard error. c) Corresponds to the cluster (interaction) in panel $\mathbf{b}$, averaged across time $( \pm \mathrm{SE})$. Whereas successful (vs unsuccessful) learning was associated with beta desynchronization after sleep, no significant difference in beta power emerged from the same contrast after sleep deprivation. d) Topographical representations of the change in beta power for remembered and forgotten pairings in the sleep and sleep deprivation conditions, averaged across the time-window of interest (0.3-2s). Colour bars represent beta power change from baseline (\%). 


\section{Discussion}

Sleep provides a benefit over wake for retaining memories and also for learning new ones (Gais et al., 2006; Ashton et al., 2020; Payne et al., 2012; Talamini et al., 2008; Gaskell et al., 2018; Cairney et al., 2018a; Durrant et al., 2016; Ashton \& Cairney, 2021; Yoo et al., 2007; Tempesta et al., 2016; Alberca-Reina et al., 2014; Kaida et al., 2015; Cousins et al., 2018). Some suggest that these benefits can be explained by an active role of sleep, in particular, slow oscillation activity (SOA), in shifting memory dependency from hippocampus to neocortex, and thus increasing hippocampal capacity for new learning (Born \& Wilhelm, 2012; Rasch \& Born, 2013; Klinzing et al., 2019). In the current study, we tested the hypothesis that overnight consolidation would predict next-day learning and that SOA would contribute to this relationship. Although we observed a benefit of sleep (relative to sleep deprivation) on our measures of overnight consolidation and next-day learning, we found no evidence of a relationship between the two measures, nor with SOA. Given the importance of sleep for new learning, we further sought to understand how sleep deprivation affects the neurocognitive mechanisms underpinning successful encoding. Interestingly, whereas learning of subsequently remembered (vs forgotten) associations was associated with a downregulation of 12-20 Hz beta power after sleep (as reported in previous work; Griffiths et al., 2019; HansImayr et al., 2009; 2011), no significant between-condition difference in beta power was observed after sleep deprivation. These findings suggest that protracted sleep loss disrupts the neural operations underpinning memory encoding, leading to suboptimal performance.

\section{Sleep benefits overnight consolidation and next-day learning}

Previous work has shown that sleep (vs wakefulness) supports memory consolidation (Gais et al., 2006; Ashton et al., 2020; Payne et al., 2012; Talamini et al., 2008; Gaskell et al., 2018; Cairney et al., 2018a; Durrant et al., 2016; Ashton \& Cairney, 2021) and subsequent learning (Cousins et al., 2018; Kaida et al., 2015; McDermott et al., 2003; Saletin et al., 2016; Tempesta et al., 2016; Yoo et al., 2007). Consistent with these studies, we found that memory retention and next-day learning were better after overnight sleep than wakefulness. One might argue that between-condition differences in retrieval and encoding performance were simply due to the increased fatigue and decreased motivation that typically accompanies prolonged sleep deprivation. However, given that the tests used to assess memory consolidation and next-day learning included provision for recovery sleep (i.e. participants were assessed $48 \mathrm{~h}$ after the overnight retention interval), it is unlikely that our results can be explained by tiredness and motivational factors alone. Relatedly, although participants were less alert after sleep 
deprivation than sleep (as indicated performance on the psychomotor vigilance task and scores on the Stanford Sleepiness Scale), subsidiary analyses showed that between-condition differences in next-day learning could not be fully accounted by these alertness measures.

Sleep-associated memory processing is thought to unfold during slow-wave sleep (SWS), such that slow oscillations and sleep spindles support reorganisation of newly acquired memory traces from hippocampus to neocortex (Born \& Wilhelm, 2012; Rasch \& Born, 2013; Klinzing et al., 2019). Yet, we observed no significant relationships between candidate sleep parameters (SOA, spindle power and time in SWS) and our index of memory consolidation. This is in line with recent work suggesting that the relationship between SWS (and its cardinal neural oscillations) and memory consolidation may not be as clear-cut as previously thought (Cordi \& Rasch, 2021).

\section{No link between sleep-associated consolidation and next-day learning}

If hippocampal-to-neocortical memory transfer during SWS paves the way for new learning in hippocampus, sleep-associated consolidation should predict next-day learning, and SOA should facilitate this relationship. However, we observed no such effects in our data, irrespective of whether our analyses included both the sleep and sleep deprivation conditions or only the sleep condition. Therefore, it seems that new learning may not be contingent on memory processing during the preceding night of sleep.

An alternative interpretation of these findings is that our paradigm could not provide an adequate test of our hypothesis. Although our consolidation (visuospatial memory) and encoding (paired associates learning) tasks are both dependent on hippocampus (Eichenbaum 2004; Konkel \& Cohen, 2009), qualitative differences between these tasks might have negated our ability to detect a relationship between sleep-associated memory processing and nextday learning. Future work can address this possibility through the use of more similar experimental measures (e.g. using paired associates learning to assess both memory retention and encoding).

\section{Sleep deprivation disrupts the neurocognitive mechanisms of learning}

Successful learning is associated with left-lateralised beta desynchronisation $\sim 0.5-1.5 \mathrm{~s}$ after stimulus onset (Griffiths et al., 2016; 2019; Hanslmayr et al., 2009; 2011; 2012; 2014). Consistent with these findings, we observed a decrease in beta power $\sim 0.3-2 \mathrm{~s}$ after stimulus 
onset for subsequently remembered (vs forgotten) associations, and this was most pronounced in the left hemisphere. Beta desynchronization is thought to reflect semantic processing during successful memory formation (Hanslmayr et al., 2011; Fellner et al., 2013; 2019); as beta power decreases, the depth of semantic processing during episodic memory formation increases (Hanslmayr et al., 2009). During our learning task, participants were instructed to form vivid mental images or stories that linked the adjective and image of each pairing. Our observed downregulation of beta power during successful learning might thus reflect the engagement of semantic processing operations as novel associations are committed to memory.

Importantly, however, the change in beta power that accompanied successful learning differed according to whether participants had slept or remained awake across the overnight interval. Whereas successful learning was associated with beta desynchronization after sleep, no significant change in beta power emerged for subsequently remembered (vs forgotten) adjective-image pairings after sleep deprivation. Hence, a protracted lack of sleep appeared to disrupt semantic processing when participants were forming new memories. This interpretation is in line with previous behavioural findings where sleep deprived individuals have difficulty encoding semantically incongruent stimulus pairs (Alberca-Reina et al., 2014). The sleep-deprived brain might thus rely on alternative processing routes when committing new information to memory. Indeed, prior studies have shown that sleep deprivation leads to compensatory neural responses during learning (Chee et al., 2004; Drummond et al., 2004) and recognition (Sterpenich et al., 2007).

What might be the nature of this alternative route to learning after sleep deprivation? It is interesting to note that we observed an upregulation of beta activity during successful (vs unsuccessful) learning after sleep deprivation (although this difference did not survive a Bonferroni correction for multiple comparisons). Increases in beta power have been linked to working memory and active rehearsal (Tallon-Baudry et al., 2001; Onton et al., 2005; Hwang et al., 2005; Deiber et al., 2007), suggesting that the brain may engage in a more surface-based rehearsal of new information when semantic processing pathways (related to beta desynchronsation) are compromised by sleep deprivation. 


\section{Conclusions}

We investigated whether sleep-associated memory consolidation would predict next-day learning and whether SOA would facilitate this relationship. Furthermore, we investigated how the neurocognitive mechanisms of learning are affected by sleep deprivation. Although sleep (vs sleep deprivation) improved memory retention and next-day learning, we found no evidence of a relationship between these measures, nor with SOA. Whereas beta desynchronization - a marker of semantic processing during episodic memory formation was intact after sleep, no learning-related change in beta power was observed after sleep deprivation. Protracted sleep loss might therefore disrupt our ability to draw upon semantic knowledge when encoding novel associations, necessitating the use of surface-based and ultimately suboptimal routes to learning

\section{Materials and Methods}

\section{Participants}

Fifty-nine participants ( 32 females, mean \pm SD age $=20.10 \pm 1.80$ ) were recruited on a voluntary basis. Twenty-nine individuals were withdrawn due to being unable to commit to the study schedule $(n=18)$; not meeting the performance criterion in the preliminary session ( $n=10$, see below) and not meeting the study requirement of being a native English speaker $(n=1)$. Our final sample size was $N=30$ participants ( 17 females, mean \pm SD age, $20.15 \pm 1.56$ ), each of whom completed both the sleep and sleep deprivation conditions (order counterbalanced). Participants reported no history sleep or psychiatric disorders and agreed to refrain from caffeine and alcohol for $24 \mathrm{~h}$ and $48 \mathrm{~h}$, respectively, before each study session. Written informed consent was obtained from all participants in line with the requirements of Research Ethics Committee of the Department of Psychology at the University of York. Participants received $£ 100$ compensation upon completion of the study.

Statistical power was calculated prior to data collection using an effect size of $d=0.56$ from Ashton et al. (2020). This effect size was derived from a paired-samples t-test comparing forgetting after a night of sleep or total sleep deprivation. Based on this effect size, our preregistered sample of $\mathrm{N}=30$ participants provided $83.7 \%$ power (alpha=.05, two-tailed). 


\section{Tasks and Stimuli}

\section{Visuospatial task}

One-hundred images of neutral scenes were taken from the International Affective Picture System (Lang et al., 1997) and the Nencki Affective Picture System (Marchewka et al., 2014). These were divided into two sets of 50 images for use in the sleep and sleep deprivation conditions (assignment of set to condition was counterbalanced). The visuospatial task was divided into three phases:

\section{Training I: Passive Viewing}

Each of 50 images was presented in a randomly-selected location on a grid background (exposure time $=3 \mathrm{~s}$, interstimulus interval $[I S I]=1 \mathrm{~s}$ ). Participants were instructed to try and memorise the image locations for a later test.

\section{Training II: Active Viewing}

Each image appeared in the centre of the grid and participants moved it to the location that they believed it had appeared at passive viewing. The image then reappeared in its correct location to serve as feedback. This continued until all images had been placed $<4.8 \mathrm{~cm}(<150$ pixels) from their correct location on two consecutive rounds of active viewing (images for which this criterion was met were dropped from subsequent active viewing rounds).

\section{Test}

The test phase followed the same procedures as one round of active viewing, with the exception that no feedback was provided. Three tests were completed (immediate, delayed and follow-up).

\section{Paired-associates task}

Two hundred images of natural and manmade objects on a white background were taken from Konkle et al. (2010) and online resources. These were divided into two sets of 100 objects (50 natural and 50 manmade) for use in the sleep and sleep deprivation conditions (assignment of set to condition was counterbalanced). Three hundred adjectives (150 adjectives per condition, assignment counterbalanced) were taken from Cairney et al. (2018). Within each condition, 100 adjectives were randomly selected as targets and the remaining 50 as foils. 


\section{Adjective familiarisation}

Each of the 100 target adjectives was presented for $3 \mathrm{~s}$. Participants were instructed to rate how often they would use each adjective in conversation on a scale of 1 to 9 ( $1=$ never, $5=$ sometimes and $9=$ often) within an additional $4 \mathrm{~s}$ (ISI with fixation crosshair $=1.5 \mathrm{~s} \pm 100 \mathrm{~ms}$ ).

2. Image familiarisation

Each of the 100 images (50 natural and 50 manmade objects) was presented for $3 \mathrm{~s}$. Participants were instructed to imagine themselves interacting with each object and then categorise it as being natural or manmade within an additional $4 \mathrm{~s}$ (ISI with fixation crosshair $=1.5 \mathrm{~s} \pm 100 \mathrm{~ms})$.

\section{Learning}

On each trial, participants were presented with an adjective and image from each of the prior familiarisation phases for $4.5 \mathrm{~s}$ and instructed to memorise the adjective-image pairing for a future test. To facilitate learning, participants were asked to create a story or mental image in their mind that involved the adjective and image interacting for the duration of the trial, and then rate this association as realistic or bizarre within an additional $4 \mathrm{~s}$. A longer ISI of $5 \mathrm{~s}$ ( $\pm 100 \mathrm{~ms}$ ) was employed to facilitate EEG analyses of adjective-image learning (this comprised a $2 \mathrm{~s}$ progression bar followed by $3 \mathrm{~s}$ fixation).

\section{Test}

Each of 150 adjectives (100 from learning and 50 unseen foils) was presented for $3 \mathrm{~s}$. Participants were first instructed to indicate whether the adjective was old or new within an additional $10 \mathrm{~s}$. Feedback on accuracy (correct/incorrect) was then provided for $1 \mathrm{~s}$. For correct old responses, participants were presented with four images (all of which had been seen at learning) and asked to indicate which image was paired with the adjective within 10 s. Participants then rated how confident they were in their response on a scale of 1 (not confident) to 4 (very confident) within 10s. For incorrect old responses or new responses, participants moved immediately onto the next trial (ISI with fixation crosshair $=1.5 \mathrm{~s} \pm 100 \mathrm{~ms}$ ).

\section{Psychomotor vigilance task (PVT)}

The PVT was obtained from Khitrov et al. (2014, bhsai.org/downloads/pc-pvt). Participants were instructed to respond as soon as a digital counter appeared on the screen (ISI $=2-10 \mathrm{~s}$ ). Participants received feedback on their response times and the task lasted for 3 min. 


\section{Procedure}

Preliminary session

Participants completed a preliminary memory assessment before entering the main study. They learned 180 semantically-related word pairs (e.g. Horizon - Sun) and were immediately tested with a cued recall procedure. Participants scoring between $50 \%$ and $95 \%$ were invited back for the main experiment. This ensured that participants were unlikely to perform at floor or ceiling in the visuospatial and paired-associates tasks.

\section{Session one: evening}

Figure 1 illustrates the experimental protocol for the main study. Participants arrived between 8:30 PM and 10 PM and individuals in the sleep condition (earlier arrivals) were immediately wired-up for overnight EEG monitoring. Participants began the study by completing the Stanford Sleepiness Scale (Hoddes et al., 1973), PVT and then the training and test phases of the visuospatial task.

\section{Overnight interval}

Participants in the sleep condition went to bed at $\sim 11 \mathrm{PM}$ and were awoken in the morning at 7 AM (thus achieving 8 $\mathrm{h}$ of EEG-monitored sleep). Participants in the sleep deprivation condition remained awake across the entire night under the supervision of a researcher. They were provided with refreshments and were permitted to play games, watch movies or complete coursework.

\section{Session two: morning}

Participants in the sleep deprivation condition were wired-up for EEG monitoring (this was not necessary in the sleep condition as electrodes had already been attached the previous night). Participants then completed another round of the Stanford Sleepiness Scale and PVT, and another (delayed) visuospatial test. Afterwards, participants carried out the familiarisation phases of the paired-associates task, before completing the paired-associates learning phase with EEG monitoring.

\section{Session three: follow-up}

Participants returned $48 \mathrm{~h}$ after session two (thereby allowing for recovery sleep in the sleep deprivation condition) and completed a final round of the Stanford Sleepiness Scale and the PVT. They then carried out the paired-associates test and a final (follow-up) visuospatial test. 


\section{Equipment}

\section{Experimental tasks}

All tasks were executed on a Windows PC and participant responses were recorded with the keyboard or mouse. The visuospatial task was implemented in Presentation version 14.1 (Neurobehavioural Systems, Inc.) whereas the paired-associates task was implemented in Psychtoolbox 3.0.13 (Brainard, 1997; Pelli, 1997; Kleiner et al., 2007) and MATLAB 2018a (The MathWorks, Inc.).

\section{EEG}

EEG recordings were administered with Embla N7000/NDx systems and REMLogic 3.4 software. Gold-plated electrodes were attached to the scalp according to the international 10-20 system at frontal (F3 and F4), central (C3 and C4), parietal (P3 and P4) and occipital (O1 and 02 ) locations, and referenced to the linked mastoids. Left and right electrooculography electrodes were attached, as were electromyography electrodes at the mentalis and submentalis bilaterally, and a ground electrode was attached to the forehead. An additional reference electrode was placed at Cz for the NDx system. Each electrode had a connection impedance of $<5 \mathrm{k} \Omega$ and all online signals were digitally sampled at $200 \mathrm{~Hz}$ (N7000) or $256 \mathrm{~Hz}$ (NDx, down-sampled to $200 \mathrm{~Hz}$ during preprocessing).

\section{Data analyses}

\section{Behaviour}

Behavioural data were analysed using R Studio (v.1.4.1717, R Core Team, 2020). Memory consolidation was indexed by the change in visuospatial memory accuracy between the immediate and delayed test. For each test, we computed an error score for each image by calculating the distance $(\mathrm{cm})$ between the recalled location (image centre) and the location that the image had appeared at passive viewing. We derived a retention index (RI) by subtracting the error score at the delayed test from the error score at the immediate test for each image, and then averaging across images (higher $\mathrm{RI}=$ better retention). A follow-up RI was calculated between the immediate and follow-up tests using the same method. One participant was removed from analyses that included $\mathrm{RI}^{\text {SleepBenefit }}$ scores because their $\mathrm{RI}$ at the delayed test in the sleep deprivation condition was >3 SD from the mean (as pre-registered). Next-day learning performance was indexed by the learning index (LI), which equated to the $\%$ of correctly recognised images on the paired-associates test. Between-group differences in $\mathrm{RI}$ and LI were analysed using paired-samples t-tests with a significance threshold at $p<.05$. 
One of our primary aims was to investigate the relationship between sleep-associated consolidation and next-day learning, and how SOA contributes to this relationship. To do this, we first quantified the benefit of sleep (vs sleep deprivation) on the RI and LI. We subtracted (for each participant) the RI in the sleep deprivation condition from the RI in the sleep

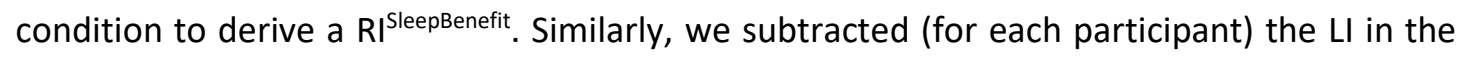
sleep deprivation condition from the $\mathrm{LI}$ in the sleep condition to obtain a LIleepBenefit. Positive scores on the $\mathrm{RI}^{\text {sleepBenefit }}$ and $\mathrm{LCl}^{\text {sleepBenefit }}$ metrics therefore indicated a sleep-associated improvement in performance. $\mathrm{RI}^{\text {sleepBenefit }}$ and SOA (see below) were entered as predictors of

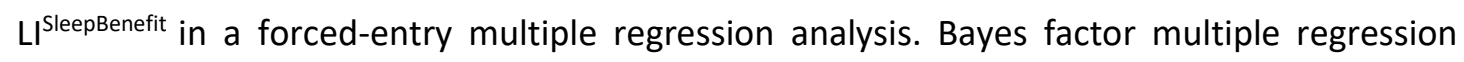
analysis (R package: BayesFactor; Morey, 2019) was used to test evidence for the null (i.e. no relationship between sleep-associated consolidation $\left[\mathrm{R}^{\text {sleepBenefit }}\right]$, SOA and next-day learning [LI $\left.\left.{ }^{\text {SleepBenefit }}\right]\right)$. Exploratory correlations were computed using Pearson's R. To compare alertness across sessions, a 2 (Condition: Sleep/Sleep Deprivation) x 3 (Session: Evening/Morning/Follow-up) repeated measures ANOVA was computed on PVT response times and Stanford Sleepiness Scale scores (followed up with Bonferroni-corrected post-hoc tests, See Supplementary Results).

\section{EEG (Sleep)}

Preprocessing. Sleep EEG data were partitioned into 30 epochs and scored in RemLogic 3.4 according to standardised criteria (Iber et al., 2007). Data scored as sleep stage N2 or slowwave sleep (SWS) were exported to MATLAB 2019a using the FieldTrip toolbox (Oostenveld et al., 2011, v.10/04/18) for further analysis. Artefacts were identified and removed using FieldTrip's databrowser (mean trials rejected \pm SD, $3.5 \pm 2.85$ ), noisy channels were removed ( 4 channels across 4 participants) and two entire datasets were excluded due to excessive noise. The remaining data (28 participants) were band-pass filtered between 0.3 and $30 \mathrm{~Hz}$ using Butterworth low-pass and high-pass filters.

Power spectral analysis. Due to significant noise in the occipital channels (as a result of electrodes detaching during the night in several participants), we included only frontal (F3 and F4), central (C3 and C4) and parietal (P3 and P4) channels in the spectral analysis (similar to Ackermann et al., 2015). Using functions from the FieldTrip toolbox, artefact-free N2 and SWS epochs were applied to a Fast Fourier Transformation with a 10.24 s Hanning window and $50 \%$ overlap. EEG Power in the SOA $(0.5-4 \mathrm{~Hz})$ and fast spindle $(12.1-16 \mathrm{~Hz})$ bands was determined by averaging across the corresponding frequency bins and across channels. 


\section{EEG (Learning)}

Preprocessing. Data were high-pass filtered at $0.5 \mathrm{~Hz}$ and segmented into trials (-3s to $4.5 \mathrm{~s}$ around stimulus onset). Trials for which participants did not provide a response were removed from the analysis (mean \pm SD excluded trials, sleep: $0.1 \pm 0.45$, sleep deprivation: $5.11 \pm 7.93$, Priest et al., 2001). Electrooculogram and electrocardiogram artifacts were identified and removed using an independent components analysis and noisy channels were interpolated via a weighted-average of their nearest neighbours (as defined by FieldTrip toolbox, Oostenveld et al., 2011, 10 channels across 6 participants). Trials were visually inspected and data from two participants was removed due to excessive noise.

Time-frequency analyses. Time-frequency representations (TFRs) were calculated separately for lower $(4-30 \mathrm{~Hz})$ and higher frequencies $(30-60 \mathrm{~Hz})$. Note that the pre-registered upper bound was $120 \mathrm{~Hz}$, but because our sampling rate was $200 \mathrm{~Hz}$ the maximum frequency was lowered to $60 \mathrm{~Hz}$ to prevent aliasing. For lower frequencies, data were convolved with a 5cycle Hanning taper in in $0.5 \mathrm{~Hz}$ frequency steps and $5 \mathrm{~ms}$ time steps using adaptive windowlength (i.e. where window length decreases with increasing frequency, e.g. $1.25 \mathrm{~s}$ at $4 \mathrm{~Hz}, 1 \mathrm{~s}$ at $5 \mathrm{~Hz}$ etc). For higher frequencies, data were convolved with tapers of Slepian sequence (3 tapers), also in steps of $0.5 \mathrm{~Hz}$ and $5 \mathrm{~ms}$ and an adaptive window-length. For this latter analysis, frequency smoothing was set to 0.4 of the frequency of interest (e.g. $20 \mathrm{~Hz}$ smoothing at $50 \mathrm{~Hz}$ ). Artefact rejection was achieved with a data-driven approach, applied separately to the analyses of lower and higher frequencies: power values that exceeded the $85^{\text {th }}$ percentile across all time/frequency points and trials were removed from each participant's dataset. TFRs were converted into \% power change relative to a -400 to $-200 \mathrm{~ms}$ pre-cue baseline window. This window was chosen to mitigate baseline contamination by post-stimulus activity while preserving proximity to cue onset (note that our post-stimulus time-window of interest started at $0.3 \mathrm{~s}$, see below). Trials were divided into subsequently remembered and forgotten adjective-image pairings (based on the test phase $48 \mathrm{~h}$ later).

Statistics. TFR analyses were performed as dependent samples analyses and corrected for multiple comparisons using FieldTrip's nonparametric cluster-based permutation method (1000 randomisations). Clusters were defined by channel * time whilst averaging across the frequency dimension (separately for the theta [4-8 Hz], alpha [8-12 Hz], beta [12-20 Hz] and gamma $[40-60 \mathrm{~Hz}]$ bands, cluster threshold $p<.05)$. Pre-registered analyses in theta and gamma bands were one-tailed, whereas exploratory analyses were two-tailed. To reduce 
interference from early visual evoked response, the time window of interest was set from 0.32s (Friedman \& Johnson, 2000; Osipova et al., 2006). A factorial approach was used to assess the impacts of sleep deprivation (vs sleep) on the neurocognitive mechanisms of encoding: we calculated the grand average TFR difference for subsequently remembered $>$ forgotten adjective-image pairings within each condition (sleep and sleep deprivation), and then entered these contrasts into the cluster-based permutation analysis (Sleep ${ }^{\text {remembered }>\text { forgotten }}>$ Sleep Deprivation $\left.{ }^{\text {remembered }>\text { forgotten }}\right)$. Cohen's $d$ effect sizes were based on averages of the largest clusters.

\section{Acknowledgements}

We are grateful to Marcus $\mathrm{O}$. Harrington for help with data collection and Jennifer E. Ashton for help with the experimental materials. We also thank members of the Sleep, Language and Memory Lab at the University of York for fruitful discussions of the data. This work was supported by a Medical Research Council Career Development Award (MR/P020208/1) to S.A.C and a University of York Department of Psychology Doctoral Studentship to A.áV.G. Study data and analysis scripts can be retrieved via the following link: osf.io/cy2s9.

\section{Competing Interests}

The authors declare no financial or non-financial competing interests.

\section{References}

Ackermann, S., Hartmann, F., Papassotiropoulos, A., de Quervain, D. J.-F., \& Rasch, B. (2015). No Associations between Interindividual Differences in Sleep Parameters and Episodic Memory Consolidation. Sleep, 38(6), 951-959. https://doi.org/10.5665/sleep.4748

Alberca-Reina, E., Cantero, J. L., \& Atienza, M. (2014). Semantic congruence reverses effects of sleep restriction on associative encoding. Neurobiology of Learning and Memory, 110, 27-34. https://doi.org/10.1016/j.nlm.2014.01.012

Antonenko, D., Diekelmann, S., Olsen, C., Born, J., \& Mölle, M. (2013). Napping to renew learning capacity: enhanced encoding after stimulation of sleep slow oscillations. The 
European Journal of Neuroscience, 37(7), 1142-1151.

https://doi.org/10.1111/ejn.12118

Ashton, J. E., \& Cairney, S. A. (2021). Future-relevant memories are not selectively

strengthened during sleep. PloS One, 16(11), e0258110.

https://doi.org/10.1371/journal.pone.0258110

Ashton, J. E., Harrington, M. O., Langthorne, D., Ngo, H.-V. V., \& Cairney, S. A. (2020). Sleep deprivation induces fragmented memory loss. Learning \& Memory , 27(4), 130-135. https://doi.org/10.1101//m.050757.119

Backhaus, J., Junghanns, K., Born, J., Hohaus, K., Faasch, F., \& Hohagen, F. (2006). Impaired declarative memory consolidation during sleep in patients with primary insomnia: Influence of sleep architecture and nocturnal cortisol release. Biological Psychiatry, 60(12), 1324-1330. https://doi.org/10.1016/j.biopsych.2006.03.051

Becker, S. P., Jarrett, M. A., Luebbe, A. M., Garner, A. A., Burns, G. L., \& Kofler, M. J. (2018). Sleep in a large, multi-university sample of college students: sleep problem prevalence, sex differences, and mental health correlates. Sleep Health, 4(2), 174-181.

https://doi.org/10.1016/j.sleh.2018.01.001

Bonnet, M. H., \& Arand, D. L. (1995). We are chronically sleep deprived. Sleep, 18(10), 908911. https://doi.org/10.1093/sleep/18.10.908

Born, J., \& Wilhelm, I. (2012). System consolidation of memory during sleep. Psychological Research, 76(2), 192-203. https://doi.org/10.1007/s00426-011-0335-6

Brainard, D. H. (1997). The Psychophysics Toolbox. Spatial Vision, 10(4), 433-436. https://www.ncbi.nlm.nih.gov/pubmed/9176952

Cairney, S. A., Guttesen, A. Á. V., El Marj, N., \& Staresina, B. P. (2018). Memory Consolidation Is Linked to Spindle-Mediated Information Processing during Sleep. Current Biology: CB, 28(6), 948-954.e4. https://doi.org/10.1016/j.cub.2018.01.087 
Cairney, S. A., Lindsay, S., Paller, K. A., \& Gaskell, M. G. (2018). Sleep preserves original and distorted memory traces. Cortex; a Journal Devoted to the Study of the Nervous System and Behavior, 99, 39-44. https://doi.org/10.1016/j.cortex.2017.10.005

Chee, M. W. L., \& Choo, W. C. (2004). Functional imaging of working memory after $24 \mathrm{hr}$ of total sleep deprivation. The Journal of Neuroscience: The Official Journal of the Society for Neuroscience, 24(19), 4560-4567. https://doi.org/10.1523/JNEUROSCI.000704.2004

Cordi, M. J., \& Rasch, B. (2021). No evidence for intra-individual correlations between sleepmediated declarative memory consolidation and slow-wave sleep. Sleep, 44(8). https://doi.org/10.1093/sleep/zsab034

Cousins, J. N., Sasmita, K., \& Chee, M. W. L. (2018). Memory encoding is impaired after multiple nights of partial sleep restriction. Journal of Sleep Research, 27(1), 138-145. https://doi.org/10.1111/jsr.12578

Cox, R., Hofman, W. F., \& Talamini, L. M. (2012). Involvement of spindles in memory consolidation is slow wave sleep-specific. Learning \& Memory, 19(7), 264-267. https://doi.org/10.1101//m.026252.112

Deiber, M.-P., Missonnier, P., Bertrand, O., Gold, G., Fazio-Costa, L., Ibañez, V., \& Giannakopoulos, P. (2007). Distinction between perceptual and attentional processing in working memory tasks: a study of phase-locked and induced oscillatory brain dynamics. Journal of Cognitive Neuroscience, 19(1), 158-172. https://doi.org/10.1162/jocn.2007.19.1.158

Drummond, S. P. A., Brown, G. G., Salamat, J. S., \& Gillin, J. C. (2004). Increasing task difficulty facilitates the cerebral compensatory response to total sleep deprivation. Sleep, 27(3), 445-451. https://www.ncbi.nlm.nih.gov/pubmed/15164897 
Durrant, S. J., Cairney, S. A., \& Lewis, P. A. (2016). Cross-modal transfer of statistical information benefits from sleep. Cortex; a Journal Devoted to the Study of the Nervous System and Behavior, 78, 85-99. https://doi.org/10.1016/j.cortex.2016.02.011

Eichenbaum, H. (2004). Hippocampus: cognitive processes and neural representations that underlie declarative memory. Neuron, 44(1), 109-120. https://doi.org/10.1016/j.neuron.2004.08.028

Fellner, M.-C., Bäuml, K.-H. T., \& HansImayr, S. (2013). Brain oscillatory subsequent memory effects differ in power and long-range synchronization between semantic and survival processing. Neurolmage, 79, 361-370. https://doi.org/10.1016/j.neuroimage.2013.04.121

Fellner, M.-C., Gollwitzer, S., Rampp, S., Kreiselmeyr, G., Bush, D., Diehl, B., Axmacher, N., Hamer, H., \& Hanslmayr, S. (2019). Spectral fingerprints or spectral tilt? Evidence for distinct oscillatory signatures of memory formation. PLoS Biology, 17(7), e3000403. https://doi.org/10.1371/journal.pbio.3000403

Friedman, D., \& Johnson, R., Jr. (2000). Event-related potential (ERP) studies of memory encoding and retrieval: A selective review. Microscopy Research and Technique, 51(1), $6-28$.

Gais, S., Lucas, B., \& Born, J. (2006). Sleep after learning aids memory recall. Learning \& Memory , 13(3), 259-262. https://doi.org/10.1101/Im.132106

Gaskell, M. G., Cairney, S. A., \& Rodd, J. M. (2018). Contextual priming of word meanings is stabilized over sleep. Cognition, 182, 109-126. https://doi.org/10.1016/j.cognition.2018.09.007

Griffiths, B. J., Mayhew, S. D., Mullinger, K. J., Jorge, J., Charest, I., Wimber, M., \& Hanslmayr, S. (2019). Alpha/beta power decreases track the fidelity of stimulus-specific information. eLife, 8. https://doi.org/10.7554/eLife.49562 
Griffiths, B. J., Parish, G., Roux, F., Michelmann, S., van der Plas, M., Kolibius, L. D., Chelvarajah, R., Rollings, D. T., Sawlani, V., Hamer, H., Gollwitzer, S., Kreiselmeyer, G., Staresina, B., Wimber, M., \& Hanslmayr, S. (2019). Directional coupling of slow and fast hippocampal gamma with neocortical alpha/beta oscillations in human episodic memory. Proceedings of the National Academy of Sciences of the United States of America, 116(43), 21834-21842. https://doi.org/10.1073/pnas.1914180116

Griffiths, B., Mazaheri, A., Debener, S., \& Hanslmayr, S. (2016). Brain oscillations track the formation of episodic memories in the real world. Neurolmage, 143, 256-266. https://doi.org/10.1016/j.neuroimage.2016.09.021

HansImayr, S., Matuschek, J., \& Fellner, M.-C. (2014). Entrainment of prefrontal beta oscillations induces an endogenous echo and impairs memory formation. Current Biology: $C B, 24(8)$, 904-909. https://doi.org/10.1016/j.cub.2014.03.007

HansImayr, S., Spitzer, B., \& Bäuml, K.-H. (2009). Brain oscillations dissociate between semantic and nonsemantic encoding of episodic memories. Cerebral Cortex , 19(7), 1631-a1640. https://doi.org/10.1093/cercor/bhn197

HansImayr, S., Staudigl, T., \& Fellner, M.-C. (2012). Oscillatory power decreases and longterm memory: the information via desynchronization hypothesis. Frontiers in Human Neuroscience, 6, 74. https://doi.org/10.3389/fnhum.2012.00074

HansImayr, S., Volberg, G., Wimber, M., Raabe, M., Greenlee, M. W., \& Bäuml, K.-H. T. (2011). The Relationship between Brain Oscillations and BOLD Signal during Memory Formation: A Combined EEG-fMRI Study. The Journal of Neuroscience: The Official Journal of the Society for Neuroscience, 31(44), 15674-15680. https://doi.org/10.1523/JNEUROSCI.3140-11.2011

Henin, S., Shankar, A., Hasulak, N., Friedman, D., Dugan, P., Melloni, L., Flinker, A., Sarac, C., Fang, M., Doyle, W., Tcheng, T., Devinsky, O., Davachi, L., \& Liu, A. (2019). Hippocampal gamma predicts associative memory performance as measured by acute and chronic 
intracranial EEG. Scientific Reports, 9(1), 593. https://doi.org/10.1038/s41598-018-

37561-z

Hoddes, E., Zarcone, V., Smythe, H., Phillips, R., \& Dement, W. C. (1973). Quantification of sleepiness: a new approach. Psychophysiology, 10(4), 431-436.

https://doi.org/10.1111/j.1469-8986.1973.tb00801.x

Hwang, G., Jacobs, J., Geller, A., Danker, J., Sekuler, R., \& Kahana, M. J. (2005). EEG correlates of verbal and nonverbal working memory. Behavioral and Brain Functions: $B B F, 1,20$. https://doi.org/10.1186/1744-9081-1-20

Iber, C. (2007). The AASM Manual for the Scoring of Sleep and Associated Events : Rules. Terminology and Technical Specification. https://ci.nii.ac.jp/naid/10024500923/

Kaida, K., Niki, K., \& Born, J. (2015). Role of sleep for encoding of emotional memory. Neurobiology of Learning and Memory, 121, 72-79. https://doi.org/10.1016/j.nlm.2015.04.002

Khitrov, M. Y., Laxminarayan, S., Thorsley, D., Ramakrishnan, S., Rajaraman, S., Wesensten, N. J., \& Reifman, J. (2014). PC-PVT: a platform for psychomotor vigilance task testing, analysis, and prediction. Behavior Research Methods, 46(1), 140-147. https://doi.org/10.3758/s13428-013-0339-9

Kleiner, M., Brainard, D., \& Pelli, D. (2007). What's new in Psychtoolbox-3? https://pure.mpg.de/rest/items/item_1790332/component/file_3136265/content

Klinzing, J. G., Niethard, N., \& Born, J. (2019). Mechanisms of systems memory consolidation during sleep. Nature Neuroscience, 22(10), 1598-1610. https://doi.org/10.1038/s41593-019-0467-3

Konkel, A., \& Cohen, N. J. (2009). Relational memory and the hippocampus: representations and methods. Frontiers in Neuroscience, 3(2), 166-174.

https://doi.org/10.3389/neuro.01.023.2009 
Konkle, T., Brady, T. F., Alvarez, G. A., \& Oliva, A. (2010). Scene memory is more detailed than you think: the role of categories in visual long-term memory. Psychological Science, 21(11), 1551-1556. https://doi.org/10.1177/0956797610385359

Köster, M., Finger, H., Graetz, S., Kater, M., \& Gruber, T. (2018). Theta-gamma coupling binds visual perceptual features in an associative memory task. Scientific Reports, 8(1), 17688. https://doi.org/10.1038/s41598-018-35812-7

Lang, P. J., Bradley, M. M., Cuthbert, B. N., \& Others. (1997). International affective picture system (IAPS): Technical manual and affective ratings. NIMH Center for the Study of Emotion and Attention, 1, 39-58. https://www2.unifesp.br/dpsicobio/adap/instructions.pdf

Leminen, M. M., Virkkala, J., Saure, E., Paajanen, T., Zee, P. C., Santostasi, G., Hublin, C., Müller, K., Porkka-Heiskanen, T., Huotilainen, M., \& Paunio, T. (2017). Enhanced Memory Consolidation Via Automatic Sound Stimulation During Non-REM Sleep. Sleep, 40(3). https://doi.org/10.1093/sleep/zsx003

Mander, B. A., Santhanam, S., Saletin, J. M., \& Walker, M. P. (2011). Wake deterioration and sleep restoration of human learning. Current Biology: CB, 21(5), R183-R184. https://doi.org/10.1016/j.cub.2011.01.019

Marchewka, A., Zurawski, Ł., Jednoróg, K., \& Grabowska, A. (2014). The Nencki Affective Picture System (NAPS): introduction to a novel, standardized, wide-range, high-quality, realistic picture database. Behavior Research Methods, 46(2), 596-610. https://doi.org/10.3758/s13428-013-0379-1

Marshall, L., Helgadóttir, H., Mölle, M., \& Born, J. (2006). Boosting slow oscillations during sleep potentiates memory. Nature, 444(7119), 610-613. https://doi.org/10.1038/nature05278

McDermott, C. M., LaHoste, G. J., Chen, C., Musto, A., Bazan, N. G., \& Magee, J. C. (2003). Sleep deprivation causes behavioral, synaptic, and membrane excitability alterations in 
hippocampal neurons. The Journal of Neuroscience: The Official Journal of the Society for Neuroscience, 23(29), 9687-9695.

https://www.ncbi.nlm.nih.gov/pubmed/14573548

Morey, R. (n.d.). BayesFactor. Github. Retrieved July 15, 2021, from

https://github.com/richarddmorey/BayesFactor

Ngo, H.-V. V., Martinetz, T., Born, J., \& Mölle, M. (2013). Auditory closed-loop stimulation of the sleep slow oscillation enhances memory. Neuron, 78(3), 545-553. https://doi.org/10.1016/j.neuron.2013.03.006

Ong, J. L., Lau, T. Y., Lee, X. K., van Rijn, E., \& Chee, M. W. L. (2020). A daytime nap restores hippocampal function and improves declarative learning. Sleep. https://doi.org/10.1093/sleep/zsaa058

Ong, J. L., Lo, J. C., Chee, N. I. Y. N., Santostasi, G., Paller, K. A., Zee, P. C., \& Chee, M. W. L. (2016). Effects of phase-locked acoustic stimulation during a nap on EEG spectra and declarative memory consolidation. Sleep Medicine, 20, 88-97. https://doi.org/10.1016/j.sleep.2015.10.016

Ong, J. L., Patanaik, A., Chee, N. I. Y. N., Lee, X. K., Poh, J.-H., \& Chee, M. W. L. (2018). Auditory stimulation of sleep slow oscillations modulates subsequent memory encoding through altered hippocampal function. Sleep, 41(5). https://doi.org/10.1093/sleep/zsy031

Onton, J., Delorme, A., \& Makeig, S. (2005). Frontal midline EEG dynamics during working memory. Neurolmage, 27(2), 341-356. https://doi.org/10.1016/j.neuroimage.2005.04.014

Oostenveld, R., Fries, P., Maris, E., \& Schoffelen, J.-M. (2011). FieldTrip: Open source software for advanced analysis of MEG, EEG, and invasive electrophysiological data. Computational Intelligence and Neuroscience, 2011, 156869. https://doi.org/10.1155/2011/156869 
Osipova, D., Takashima, A., Oostenveld, R., Fernández, G., Maris, E., \& Jensen, O. (2006). Theta and gamma oscillations predict encoding and retrieval of declarative memory. The Journal of Neuroscience: The Official Journal of the Society for Neuroscience, 26(28), 7523-7531. https://doi.org/10.1523/JNEUROSCI.1948-06.2006

Papalambros, N. A., Santostasi, G., Malkani, R. G., Braun, R., Weintraub, S., Paller, K. A., \& Zee, P. C. (2017). Acoustic Enhancement of Sleep Slow Oscillations and Concomitant Memory Improvement in Older Adults. Frontiers in Human Neuroscience, 11, 109. https://doi.org/10.3389/fnhum.2017.00109

Payne, J. D., Tucker, M. A., Ellenbogen, J. M., Wamsley, E. J., Walker, M. P., Schacter, D. L., \& Stickgold, R. (2012). Memory for semantically related and unrelated declarative information: the benefit of sleep, the cost of wake. PloS One, 7(3), e33079. https://doi.org/10.1371/journal.pone.0033079

Pelli, D. G. (1997). The VideoToolbox software for visual psychophysics: transforming numbers into movies. Spatial Vision, 10(4), 437-442. https://www.ncbi.nlm.nih.gov/pubmed/9176953

Perl, O., Arzi, A., Sela, L., Secundo, L., Holtzman, Y., Samnon, P., Oksenberg, A., Sobel, N., \& Hairston, I. S. (2016). Odors enhance slow-wave activity in non-rapid eye movement sleep. Journal of Neurophysiology, 115(5), 2294-2302. https://doi.org/10.1152/jn.01001.2015

Priest, B., Brichard, C., Aubert, G., Liistro, G., \& Rodenstein, D. O. (2001). Microsleep during a simplified maintenance of wakefulness test. A validation study of the OSLER test. American Journal of Respiratory and Critical Care Medicine, 163(7), 1619-1625. https://doi.org/10.1164/ajrccm.163.7.2007028

R Core Team (2020). R: A language and environment for statistical computing. R Foundation for Statistical Computing, Vienna, Austria. URL https://www.R-project.org/. 
Rasch, B., \& Born, J. (2013). About sleep's role in memory. Physiological Reviews, 93(2), 681766. https://doi.org/10.1152/physrev.00032.2012

Rasch, B., Büchel, C., Gais, S., \& Born, J. (2007). Odor cues during slow-wave sleep prompt declarative memory consolidation. Science, 315(5817), 1426-1429. https://doi.org/10.1126/science.1138581

Saletin, J. M., \& Walker, M. P. (2012). Nocturnal mnemonics: sleep and hippocampal memory processing. Frontiers in Neurology, 3, 59. https://doi.org/10.3389/fneur.2012.00059

Schönauer, M., Alizadeh, S., Jamalabadi, H., Abraham, A., Pawlizki, A., \& Gais, S. (2017). Decoding material-specific memory reprocessing during sleep in humans. Nature Communications, 8, 15404. https://doi.org/10.1038/ncomms15404

Schreiner, T., Petzka, M., Staudigl, T., \& Staresina, B. P. (2021). Endogenous memory reactivation during sleep in humans is clocked by slow oscillation-spindle complexes. Nature Communications, 12(1), 3112. https://doi.org/10.1038/s41467-021-23520-2

Scullin, M. K. (2013). Sleep, memory, and aging: the link between slow-wave sleep and episodic memory changes from younger to older adults. Psychology and Aging, 28(1), 105-114. https://doi.org/10.1037/a0028830

Sterpenich, V., Albouy, G., Boly, M., Vandewalle, G., Darsaud, A., Balteau, E., Dang-Vu, T. T., Desseilles, M., D’Argembeau, A., Gais, S., Rauchs, G., Schabus, M., Degueldre, C., Luxen, A., Collette, F., \& Maquet, P. (2007). Sleep-related hippocampo-cortical interplay during emotional memory recollection. PLoS Biology, 5(11), e282. https://doi.org/10.1371/journal.pbio.0050282

Stranges, S., Tigbe, W., Gómez-Olivé, F. X., Thorogood, M., \& Kandala, N.-B. (2012). Sleep problems: an emerging global epidemic? Findings from the INDEPTH WHO-SAGE study among more than 40,000 older adults from 8 countries across Africa and Asia. Sleep, 35(8), 1173-1181. https://doi.org/10.5665/sleep.2012 
Summerfield, C., \& Mangels, J. A. (2005). Coherent theta-band EEG activity predicts itemcontext binding during encoding. Neurolmage, 24(3), 692-703. https://doi.org/10.1016/j.neuroimage.2004.09.012

Talamini, L. M., Nieuwenhuis, I. L. C., Takashima, A., \& Jensen, O. (2008). Sleep directly following learning benefits consolidation of spatial associative memory. Learning \& Memory , 15(4), 233-237. https://doi.org/10.1101//m.771608

Tallon-Baudry, C., Bertrand, O., \& Fischer, C. (2001). Oscillatory synchrony between human extrastriate areas during visual short-term memory maintenance. The Journal of Neuroscience: The Official Journal of the Society for Neuroscience, 21(20), RC177. https://www.ncbi.nlm.nih.gov/pubmed/11588207

Tamminen, J., Payne, J. D., Stickgold, R., Wamsley, E. J., \& Gaskell, M. G. (2010). Sleep spindle activity is associated with the integration of new memories and existing knowledge. The Journal of Neuroscience: The Official Journal of the Society for Neuroscience, 30(43), 14356-14360. https://doi.org/10.1523/JNEUROSCI.302810.2010

Tempesta, D., Socci, V., Coppo, M., Dello Ioio, G., Nepa, V., De Gennaro, L., \& Ferrara, M. (2016). The effect of sleep deprivation on the encoding of contextual and noncontextual aspects of emotional memory. Neurobiology of Learning and Memory, 131, 9-17. https://doi.org/10.1016/j.nlm.2016.03.007

Van Der Werf, Y. D., Altena, E., Schoonheim, M. M., Sanz-Arigita, E. J., Vis, J. C., De Rijke, W., \& Van Someren, E. J. W. (2009). Sleep benefits subsequent hippocampal functioning. Nature Neuroscience, 12(2), 122-123. https://doi.org/10.1038/nn.2253

Wang, B., Antony, J. W., Lurie, S., Brooks, P. P., Paller, K. A., \& Norman, K. A. (2019). Targeted Memory Reactivation during Sleep Elicits Neural Signals Related to Learning Content. The Journal of Neuroscience: The Official Journal of the Society for Neuroscience, 39(34), 6728-6736. https://doi.org/10.1523/JNEUROSCI.2798-18.2019 
Weisz, N., Kraft, N. G., \& Demarchi, G. (2020). Auditory cortical alpha/beta

desynchronization prioritizes the representation of memory items during a retention period. eLife, 9. https://doi.org/10.7554/eLife.55508

Yoo, S.-S., Hu, P. T., Gujar, N., Jolesz, F. A., \& Walker, M. P. (2007). A deficit in the ability to form new human memories without sleep. Nature Neuroscience, 10(3), 385-392. https://doi.org/10.1038/nn1851 